\title{
Disjoint Unit Spheres Admit At Most Two Line Transversals
}

\author{
Otfried Cheong ${ }^{1} \quad$ Xavier Goaoc ${ }^{2} \quad$ Hyeon-Suk Na ${ }^{3}$
}

May 27, 2003

\begin{abstract}
We show that a set of $n$ disjoint unit spheres in $\mathbb{R}^{d}$ admits at most two distinct geometric permutations, or line transversals, if $n$ is large enough. This bound is optimal.
\end{abstract}

\section{Introduction}

A line $\ell$ is a line transversal for a set $\mathcal{S}$ of pairwise disjoint convex bodies in $\mathbb{R}^{d}$ if it intersects every element of $\mathcal{S}$. A line transversal defines two linear orders on $\mathcal{S}$, namely the order in which $\ell$ intersects the bodies, where we can choose to orient $\ell$ in two directions. Since the two orders are essentially the same (one is the reverse of the other), we consider them as a single geometric permutation.

Bounds on the maximum number of geometric permutations were established about a decade ago: a tight bound of $2 n-2$ is known for $d=2$ [2], for higher dimension the number is in $\Omega\left(n^{d-1}\right)$ [5] and in $O\left(n^{2 d-2}\right)$ [10]. The gap was closed for the special case of spheres by Smorodinsky et al. [8], who showed that $n$ spheres in $\mathbb{R}^{d}$ admit $\Theta\left(n^{d-1}\right)$ geometric permutations. This result can be generalized to "fat" convex objects [7].

The even more specialized case of congruent spheres was treated by Smorodinsky et al. [8] and independently by Asinowski [1]. They proved that $n$ unit circles in $\mathbb{R}^{2}$ admit at most two geometric permutations if $n$ is large enough (the proof by Asinowski holds for all $n \geq 4$ ). Zhou and Suri established an upper bound of 16 for all $d$ and $n$ sufficiently large, a result quickly improved by Katchalski, Suri, and Zhou [6] and independently by Huang, Xu, and Chen [4] to 4 .

Katchalski et al. show that for $n$ large enough, two line transversals can make an angle of at most $O(1 / n)$ with each other, so all line transversals are "essentially" parallel. They define a switched pair to be a pair of spheres $(A, B)$ such that there are two line transversals $\ell$ and $\ell^{\prime}$ (for all $n$ spheres) where $\ell$ visits $A$ before $B$, while $\ell^{\prime}$ visits $B$ before $A$. Katchalski et al. prove that any sphere can participate in at most one switched pair, and that the two spheres forming a switched pair must appear consecutively in any geometric perturbation of the set. It follows that any two geometric perturbations differ only in that the elements of some switched pair may have been exchanged. Katchalski et al.'s main result is that there are at most two switched pairs in a set of $n$ disjoint unit spheres, implying the bound of four geometric permutations.

We show that in fact there cannot be more than one switched pair. This implies that, for $n$ large enough, a set of $n$ disjoint unit spheres admits at most two geometric perturbations, which differ only by the swapping of two adjacent elements. Since there are arbitrarily large sets of unit spheres in $\mathbb{R}^{d}$ with one switched pair, this bound is optimal.

The study of geometric permutations is motivated mostly by theoretical interest, yet there is an important application to computer graphics. In various applications, space is decomposed into boxes, and light can pass only through portals, that is openings between the boxes (such as doors,

\footnotetext{
${ }^{1}$ Department of Mathematics and Computer Science, TU Eindhoven, P.O. Box 513, 5600 MB Eindhoven, The Netherlands. Email: o.cheong@tue.nl

${ }^{2}$ LORIA (INRIA Lorraine), 615, rue du Jardin Botanique, B.P. 101, 54602 Villers-les-Nancy, France. Email: xavier.goaoc@loria.fr

${ }^{3}$ Soongshil University, Seoul, South Korea. Email: hsnaa@computing.ssu.ac.kr
} 


\begin{tabular}{|c|c|c|}
\hline dimension & Objects & Known bound \\
\hline \hline 2 & convex objects & $\Theta(2 n-2)[2]$ \\
\hline 2 & $n \geq 4$ congruent disks & $2[1,8]$ \\
\hline 3 & spheres with a radius disparity of $\gamma$ & $O\left(\gamma^{\log \gamma}\right)[?]$ \\
\hline any & convex objects & $\Omega\left(n^{d-1}\right)[5]$ and $O\left(n^{2 d-2}\right)[10]$ \\
\hline any & spheres of various radii, fat convex objects & $\Theta\left(n^{d-1}\right)[8,7]$ \\
\hline any & $n$ sufficiently large congruent spheres & 2 (this paper) $[6,4]$ \\
\hline \hline
\end{tabular}

Table 1: Known bounds on the number of geometric permutations.

windows, and stairwell openings in building models) [9]. For a box to be visible from another box, light has to pass through all the portals in-between, and if no line transversals for these portals exists, then the two boxes are mutually invisible.

Surveys of geometric transversal theory are Goodman et al. [3] and Wenger [11].

\section{The proof}

A unit sphere is a sphere of radius 1. We say that two unit spheres are disjoint if their interiors are (in other words, we allow the spheres to touch). A line stabs a sphere if it intersects the closed sphere (and so a tangent to a sphere stabs it). A line transversal for a set of disjoint unit spheres is a line that stabs all the spheres, with the restriction that it is not allowed to be tangent to two spheres in a common point.

Given two disjoint unit spheres $A$ and $B$, let $g(A, B)$ be their center of gravity and $\Pi(A, B)$ be their bisecting hyperplane. If the centers of $A$ and $B$ are $a$ and $b$, then $g(A, B)$ is the mid-point of $a$ and $b$, and $\Pi(A, B)$ is the hyperplane through $g(A, B)$ orthogonal to the line $a b$.

We first repeat a basic lemma by Katchalski et al.

Lemma 1 [6, Lemma 2.3] Let $\ell$ and $\ell^{\prime}$ be two different line transversals of a set $\mathcal{S}$ of $n$ disjoint unit spheres in $\mathbb{R}^{d}$. Then the angle between the direction vectors of $\ell$ and $\ell^{\prime}$ is $O(1 / n)$.

Proof. A volume argument shows that the distance between the first and last sphere stabbed by $\ell$ is $\Omega(n)$. Since $\ell$ and $\ell^{\prime}$ have distance at most 2 over an interval of length $\Omega(n)$, their direction vectors make an angle of $O(1 / n)$.

Lemma 1 implies that all line transversals for a set of spheres are nearly parallel.

We continue with a warm-up lemma in two dimensions.

Lemma 2 Let $S$ and $T$ be two unit-radius disks in $\mathbb{R}^{2}$ with centers $(-\lambda, 0)$ and $(\lambda, 0)$, where $\lambda \geq \cos \beta$ for some angle $\beta$ with $0<\beta \leq \pi / 2$. Then $S \cap T$ is contained in the ellipse

$$
\left(\frac{x}{\sin ^{2} \beta}\right)^{2}+\left(\frac{y}{\sin \beta}\right)^{2} \leq 1 \text {. }
$$

Proof. Let $(\mu, 0)$ and $(0, \nu)$ be the rightmost and topmost point of $S \cap T$ (see Figure 1). Consider the ellipse $E$ defined as

$$
\left(\frac{x}{\mu}\right)^{2}+\left(\frac{y}{\nu}\right)^{2} \leq 1
$$

$E$ intersects the boundary of $S$ in $(0, \nu)$ and $(0,-\nu)$, and is tangent to it in $(\mu, 0)$. An ellipse can intersect a circle in at most four points and the tangency counts as two intersections, and so there is no further intersection between the two curves. Since $(-\mu, 0)$ lies on $E$ and inside $S$, the part of $E$ between $(0,-\nu)$ and $(0, \nu)$ lies outside $S$, and $S \cap T$ is contained in $E$. It remains to observe that

$$
\nu^{2}=1-\lambda^{2} \leq 1-\cos ^{2} \beta=\sin ^{2} \beta,
$$




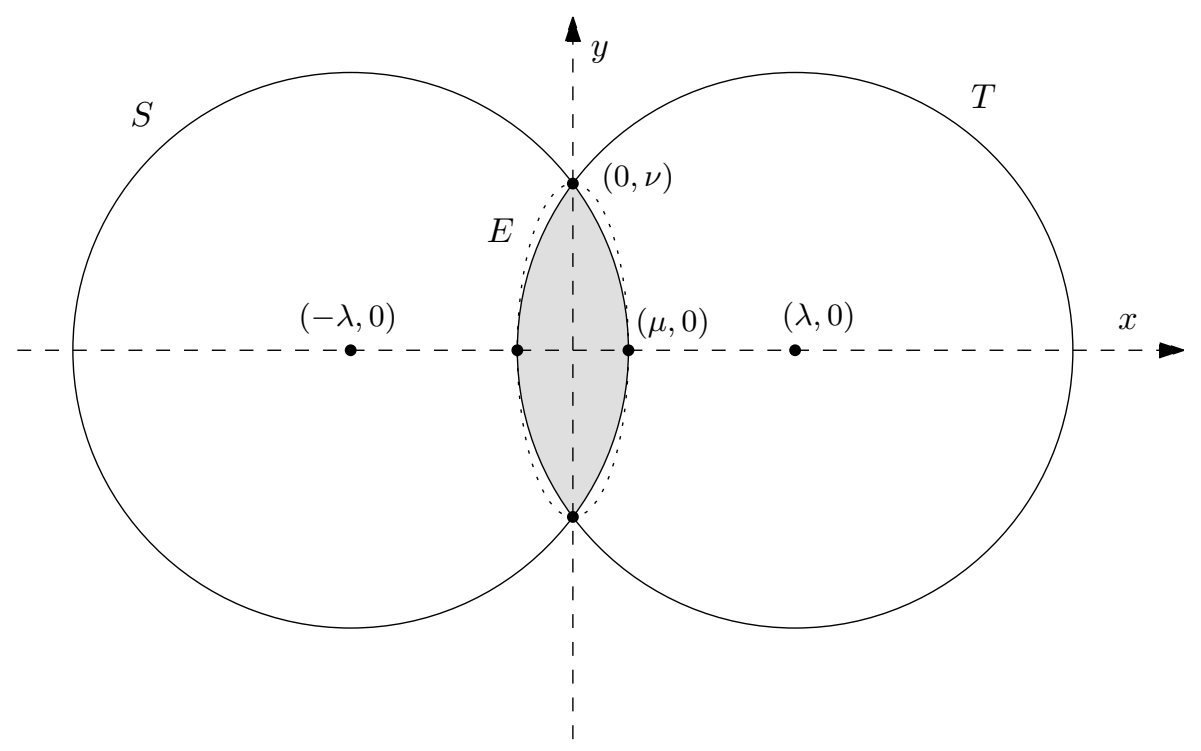

Figure 1: The intersection of two disks is contained in an ellipse.

so $\nu \leq \sin \beta$, and

$$
\mu=1-\lambda \leq 1-\cos \beta \leq 1-\cos ^{2} \beta=\sin ^{2} \beta,
$$

which proves the lemma.

We now show that a transversal for two spheres cannot pass too far from their common center of gravity.

Lemma 3 Given two disjoint unit spheres $A$ and $B$ in $\mathbb{R}^{d}$ and a line $\ell$ stabbing both spheres, let $p$ be the point of intersection of $\ell$ and $\Pi(A, B)$, and let $\beta$ be the angle between $\ell$ and $\Pi(A, B)$. Then

$$
d(p, g(A, B)) \leq \sin \beta .
$$

Proof. Let $a$ and $b$ be the centers of $A$ and $B$ and let $v$ be the direction vector of $\ell$, that is, $\ell$ can be written as $\{p+\lambda v \mid \lambda \in \mathbb{R}\}$. We first argue that proving the lemma for $d=3$ is sufficient. Indeed, assume $d>3$ and consider the 3 -dimensional subspace $\Gamma$ containing $\ell, a$, and $b$. Since we have $d(a, \ell) \leq 1$ and $d(b, \ell) \leq 1$, the line $\ell$ stabs the 3 -dimensional unit spheres $A \cap \Gamma$ and $B \cap \Gamma$. And since $\pi / 2-\beta$ is the angle between two vectors in $\Gamma$, namely $v$ and $b-a, \beta$ is also the angle between $\ell$ and the two-dimensional plane $\Pi(A, B) \cap \Gamma$. So if the lemma holds in $\Gamma$, then it also holds in $\mathbb{R}^{d}$.

In the rest of the proof we can therefore assume that $d=3$. We choose a coordinate system where $a=(0,0,-\rho), b=(0,0, \rho)$ with $\rho \geq 1$, and $v=(\cos \beta, 0, \sin \beta)$. Then $\Pi:=\Pi(A, B)$ is the $x y$-plane and $g:=g(A, B)=(0,0,0)$. Consider the cylinders $\operatorname{cyl}(A):=\{u+\lambda v \mid u \in A, \lambda \in \mathbb{R}\}$ and $\operatorname{cyl}(B)$. Since $\ell \operatorname{stabs} A$ and $B$, we have $p \in \operatorname{cyl}(A) \cap \operatorname{cyl}(B) \cap \Pi$.

The intersection $B^{\prime}:=\operatorname{cyl}(B) \cap \Pi$ is the ellipse (see Figure 2)

$$
\sin ^{2} \beta\left(x+\frac{\rho}{\tan \beta}\right)^{2}+y^{2} \leq 1,
$$

and symmetrically $A^{\prime}:=\operatorname{cyl}(A) \cap \Pi$ is

$$
\sin ^{2} \beta\left(x-\frac{\rho}{\tan \beta}\right)^{2}+y^{2} \leq 1 .
$$

If we let $\tau$ be the linear transformation

$$
\tau:(x, y) \mapsto(x \sin \beta, y),
$$




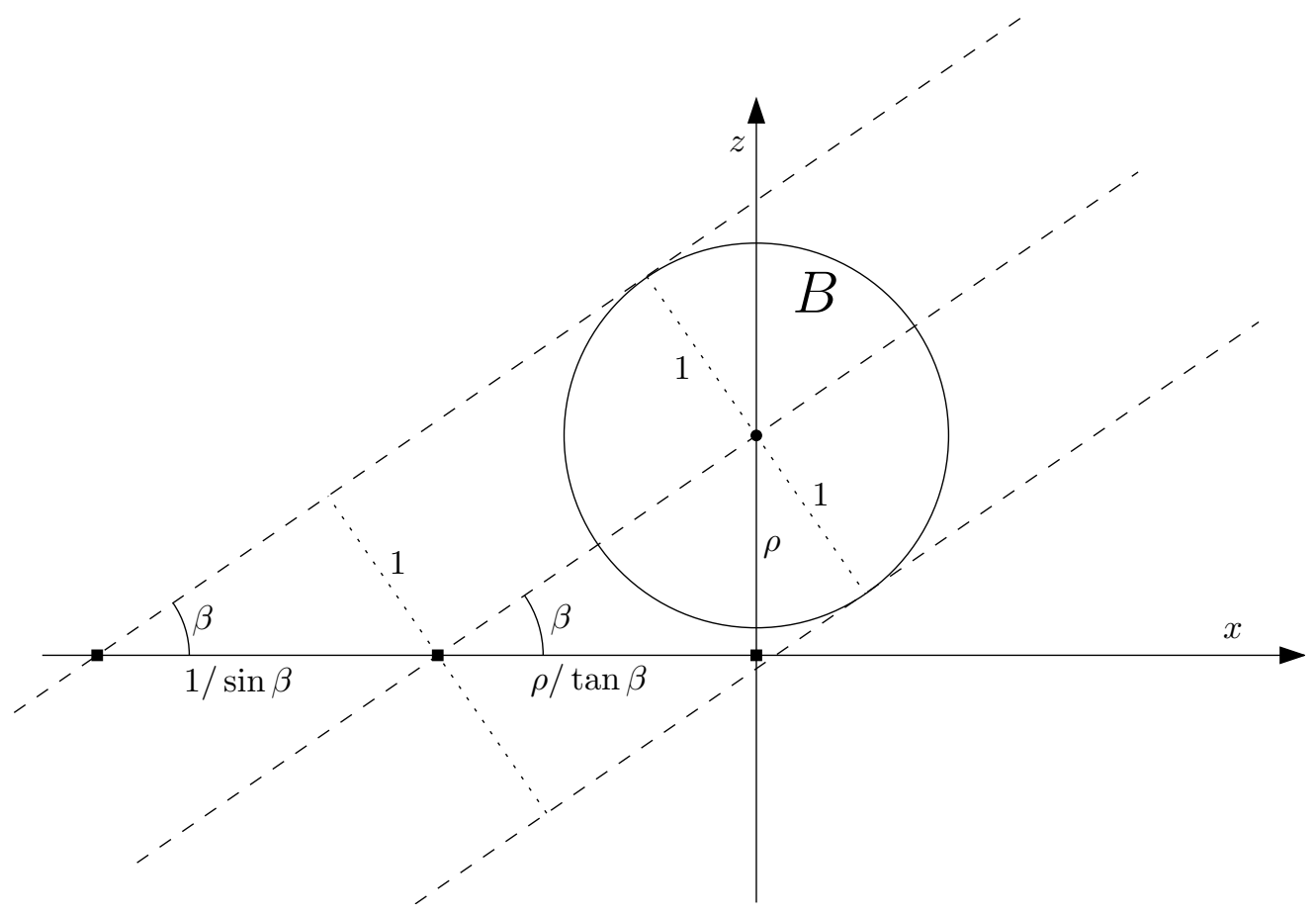

Figure 2: The intersection of the cylinder with the $x y$-plane is an ellipse.

then $\tau\left(A^{\prime}\right)$ and $\tau\left(B^{\prime}\right)$ are unit-radius disks with centers $(\rho \cos \beta, 0)$ and $(-\rho \cos \beta, 0)$. By Lemma 2 , the intersection $\tau\left(A^{\prime} \cap B^{\prime}\right)$ is contained in the ellipse

$$
\left(\frac{x}{\sin ^{2} \beta}\right)^{2}+\left(\frac{y}{\sin \beta}\right)^{2} \leq 1
$$

Applying $\tau^{-1}$ we find that $A^{\prime} \cap B^{\prime}$ is contained in the circle with radius $\sin \beta$ around $g$. Since $p \in A^{\prime} \cap B^{\prime}$, the lemma follows.

We now prove our key lemma.

Lemma 4 Let $A, B, C, D$ be four spheres from a set $\mathcal{S}$ of $n$ disjoint unit spheres in $\mathbb{R}^{d}$, for $n$ large enough. Assume there are two line transversal $\ell$ and $\ell^{\prime}$ for $\mathcal{S}$, such that $\ell$ stabs the four spheres in the order $A B C D$, and $\ell^{\prime}$ stabs them in the order $B A D C$. Then

$$
d(g(A, B), g(C, D))<1+O(1 / n) .
$$

Proof. Let $\Pi_{1}:=\Pi(A, B), \Pi_{2}=\Pi(C, D), g_{1}:=g(A, B)$, and $g_{2}:=g(C, D)$. We choose a coordinate system where $\Pi_{1}$ is the hyperplane $x_{1}=0$, and the intersection $\Pi_{1} \cap \Pi_{2}$ is the subspace $x_{1}=x_{2}=0$. We can make this choice such that the $x_{1}$-coordinate of the center of $A$ is $<0$, and that the $x_{2}$-coordinate of the center of $C$ is less than the $x_{2}$-coordinate of the center of $D$. We can also assume that the $x_{2}$-coordinate of $g_{1}$ is $\geq 0$ (otherwise we swap $A$ with $B, C$ with $D$, and $\ell$ with $\ell^{\prime}$ ). Figure 3 shows the projection of the situation on the $x_{1} x_{2}$-plane.

Let $p_{i}:=\ell \cap \Pi_{i}, p_{i}^{\prime}:=\ell^{\prime} \cap \Pi_{i}$, let $\beta_{i}$ be the angle between $\ell$ and $\Pi_{i}$, and let $\beta_{i}^{\prime}$ be the angle between $\ell^{\prime}$ and $\Pi_{i}$. By Lemma 1 we have $\beta_{i}, \beta_{i}^{\prime} \in O(1 / n)$.

Let us choose an orientation on $\ell$ and $\ell^{\prime}$ so that they intersect $\Pi_{1}$ before $\Pi_{2}$. Since $\ell$ stabs $A$ before $B$ and $C$ before $D$, it intersects $\Pi_{1}$ from bottom to top, and $\Pi_{2}$ from left to right. The segment $p_{1} p_{2}$ therefore lies in the top-left quadrant of Figure 3 . On the other hand, $\ell^{\prime}$ stabs $B$ before $A$ and $D$ before $C$, so it intersects $\Pi_{1}$ from top to bottom, and $\Pi_{2}$ from right to left, and the segment $p_{1}^{\prime} p_{2}^{\prime}$ lies in the bottom-right quadrant of the figure. 


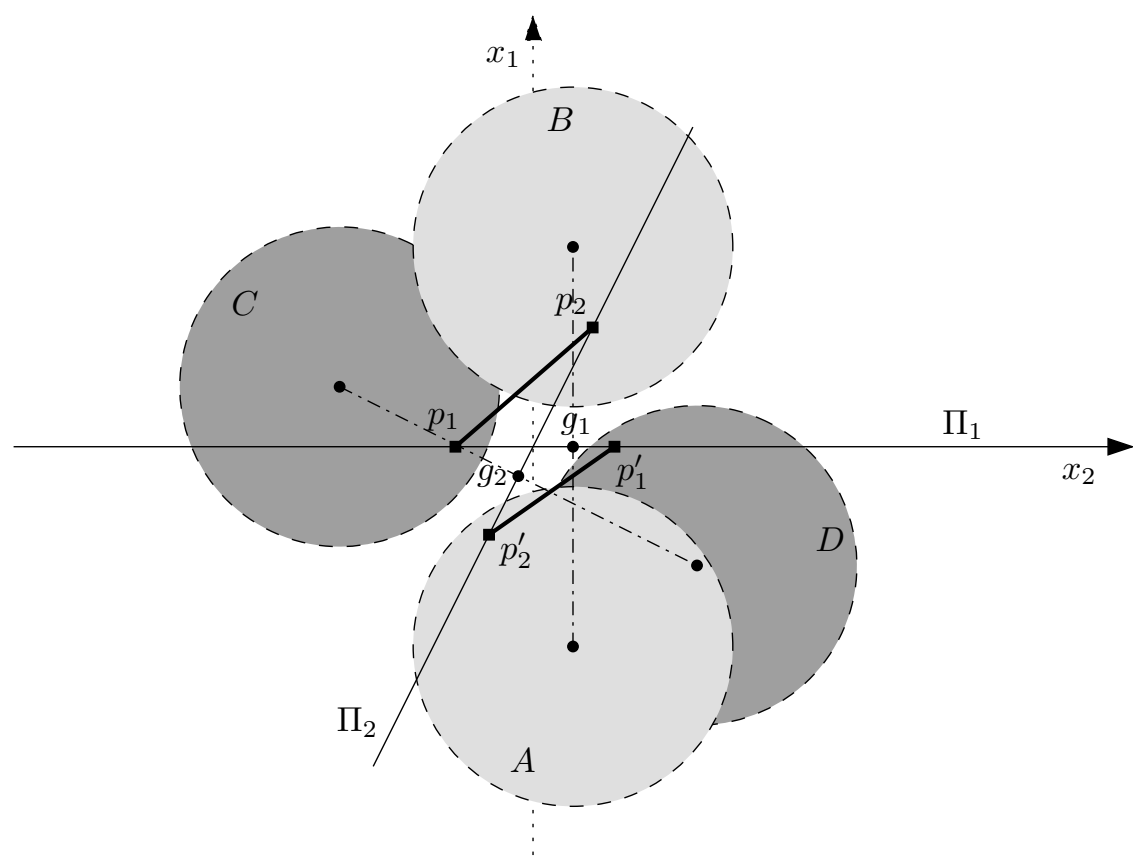

Figure 3: The two hyperplanes define four quadrants

Let now $t:=d\left(p_{1}, p_{2}\right)$ and $t^{\prime}:=d\left(p_{1}^{\prime}, p_{2}^{\prime}\right)$. Lemma 3 implies

$$
d\left(g_{1}, g_{2}\right) \leq d\left(g_{1}, p_{1}\right)+d\left(p_{1}, p_{2}\right)+d\left(p_{2}, g_{2}\right) \leq \sin \beta_{1}+t+\sin \beta_{2} \leq t+O(1 / n),
$$

and similarly

$$
d\left(g_{1}, g_{2}\right) \leq d\left(g_{1}, p_{1}^{\prime}\right)+d\left(p_{1}^{\prime}, p_{2}^{\prime}\right)+d\left(p_{2}^{\prime}, g_{2}\right) \leq \sin \beta_{1}^{\prime}+t^{\prime}+\sin \beta_{2}^{\prime} \leq t^{\prime}+O(1 / n),
$$

and so

$$
d\left(g_{1}, g_{2}\right) \leq O(1 / n)+\min \left\{t, t^{\prime}\right\} .
$$

It remains to prove that $\min \left\{t, t^{\prime}\right\} \leq 1$. Let $u_{1}\left(u_{1}^{\prime}\right)$ be the orthogonal projection of $p_{1}\left(p_{1}^{\prime}\right)$ on $\Pi_{2}$, $u_{2}\left(u_{2}^{\prime}\right)$ the orthogonal projection of $p_{2}\left(p_{2}^{\prime}\right)$ on $\Pi_{1}$. Consider the rectangular triangle $p_{1} u_{2} p_{2}$. We have $\angle u_{2} p_{1} p_{2}=\beta_{1}$, and so

$$
t \sin \beta_{1}=d\left(p_{2}, u_{2}\right)=d\left(p_{2}, \Pi_{1}\right) .
$$

Similarly, we can consider the rectangular triangles $p_{2} u_{1} p_{1}, p_{1}^{\prime} u_{2}^{\prime} p_{2}^{\prime}$, and $p_{2}^{\prime} u_{1}^{\prime} p_{1}^{\prime}$ to obtain

$$
\begin{aligned}
t \sin \beta_{2} & =d\left(p_{1}, u_{1}\right)=d\left(p_{1}, \Pi_{2}\right), \\
t^{\prime} \sin \beta_{1}^{\prime} & =d\left(p_{2}^{\prime}, u_{2}^{\prime}\right)=d\left(p_{2}^{\prime}, \Pi_{1}\right), \\
t^{\prime} \sin \beta_{2}^{\prime} & =d\left(p_{1}^{\prime}, u_{1}^{\prime}\right)=d\left(p_{1}^{\prime}, \Pi_{2}\right) .
\end{aligned}
$$

We now distinguish two cases.

The first case occurs if, as in the figure, the $x_{1}$-coordinate of $g_{2}$ is $\leq 0$. By Lemma 3 we have $d\left(p_{2}, g_{2}\right) \leq \sin \beta_{2}$. Since $p_{2}$ and $g_{2}$ lie on opposite sides of $\Pi_{1}$, we have $d\left(p_{2}, \Pi_{1}\right) \leq \sin \beta_{2}$. Similarly, we have $d\left(p_{1}, g_{1}\right) \leq \sin \beta_{1}$, and $p_{1}$ and $g_{1}$ lie on opposite sides of $\Pi_{2}$, implying $d\left(p_{1}, \Pi_{2}\right) \leq \sin \beta_{1}$. Plugging into Eq. (1) and (2), we obtain

$$
t \leq \min \left\{\frac{\sin \beta_{2}}{\sin \beta_{1}}, \frac{\sin \beta_{1}}{\sin \beta_{2}}\right\} \leq 1,
$$

which proves the lemma for this case. 
The second case occurs if the $x_{1}$-coordinate of $g_{2}$ is $>0$. We let $s_{1}:=d\left(g_{1}, \Pi_{2}\right)$, and $s_{2}:=$ $d\left(g_{2}, \Pi_{1}\right)$. Applying Lemma 3 , we then have

$$
\begin{aligned}
& d\left(p_{2}, \Pi_{1}\right) \leq d\left(p_{2}, g_{2}\right)+s_{2} \leq \sin \beta_{2}+s_{2}, \\
& d\left(p_{1}, \Pi_{2}\right) \leq d\left(p_{1}, g_{1}\right)-s_{1} \leq \sin \beta_{1}-s_{1}, \\
& d\left(p_{2}^{\prime}, \Pi_{1}\right) \leq d\left(p_{2}^{\prime}, g_{2}\right)-s_{2} \leq \sin \beta_{2}^{\prime}-s_{2}, \\
& d\left(p_{1}^{\prime}, \Pi_{2}\right) \leq d\left(p_{1}^{\prime}, g_{1}\right)+s_{1} \leq \sin \beta_{1}^{\prime}+s_{1} .
\end{aligned}
$$

Plugging Ineqs. (5) to (8) into (1) to (4), we obtain

$$
\begin{aligned}
t & \leq \frac{\sin \beta_{2}+s_{2}}{\sin \beta_{1}}, \\
t & \leq \frac{\sin \beta_{1}-s_{1}}{\sin \beta_{2}}, \\
t^{\prime} & \leq \frac{\sin \beta_{2}^{\prime}-s_{2}}{\sin \beta_{1}^{\prime}}, \\
t^{\prime} & \leq \frac{\sin \beta_{1}^{\prime}+s_{1}}{\sin \beta_{2}^{\prime}} .
\end{aligned}
$$

We want to prove that $\min \left(t, t^{\prime}\right) \leq 1$. We assume the contrary. From $t>1$ and Ineq. (10) we obtain

$$
\sin \beta_{2}<\sin \beta_{1}-s_{1},
$$

and from $t^{\prime}>1$ and Ineq. (11) we get

$$
\sin \beta_{1}^{\prime}<\sin \beta_{2}^{\prime}-s_{2} .
$$

Plugging this into Ineq. (9) and (12) results in

$$
\begin{aligned}
t & \leq \frac{\sin \beta_{2}+s_{2}}{\sin \beta_{1}}<\frac{\sin \beta_{1}-s_{1}+s_{2}}{\sin \beta_{1}}=1+\frac{s_{2}-s_{1}}{\sin \beta_{1}} \\
t^{\prime} & \leq \frac{\sin \beta_{1}^{\prime}+s_{1}}{\sin \beta_{2}^{\prime}}<\frac{\sin \beta_{2}^{\prime}-s_{2}+s_{1}}{\sin \beta_{2}^{\prime}}=1+\frac{s_{1}-s_{2}}{\sin \beta_{2}^{\prime}}
\end{aligned}
$$

It follows that if $s_{2}<s_{1}$ then $t<1$, otherwise $t^{\prime} \leq 1$. In either case the lemma follows.

Given a set $\mathcal{S}$ of $n$ spheres, Katchalski et al. [6] define a switched pair to be a pair of spheres $(A, B)$ from $\mathcal{S}$ such that there is a line transversal $\ell$ of $\mathcal{S}$ stabbing $A$ before $B$ and another line transversal $\ell^{\prime}$ of $\mathcal{S}$ stabbing $B$ before $A$. (Both transversals must be oriented in the same direction, as discussed in the remark after Lemma 1.)

The notion of switched pair is well defined because of the following lemma.

Lemma 5 [6, Lemma 2.8] Let $\mathcal{S}$ be a set of $n$ disjoint unit spheres in $\mathbb{R}^{d}$, with $n$ large enough. $A$ sphere of $\mathcal{S}$ can appear in at most one switched pair.

The number of switched pairs determines the number of geometric permutations, as the following lemma shows.

Lemma 6 [6, Lemma 2.9] Let $\mathcal{S}$ be a set of $n$ disjoint unit spheres in $\mathbb{R}^{d}$, for $n$ large enough. The two members of a switched pair must appear consecutively in in all geometric permutations of $\mathcal{S}$. If there are a total of $m$ switched pairs, then $\mathcal{S}$ admits at most $2^{m}$ different geometric permutations.

The following lemma provides a lower bound on the distance of the centers of gravity of two switched pair. It will be a key ingredient in our proof that only one switched pair can exist, as the lower bound contradicts the upper bound we have shown in Lemma 4 . 
Lemma 7 [6, Lemma 3.2] Let $\mathcal{S}$ be a set of $n$ disjoint unit spheres in $\mathbb{R}^{d}$ with two switched pairs $(A, B)$ and $(C, D)$. Then

$$
d(g(A, B), g(C, D)) \geq \sqrt{2}-\varepsilon(n),
$$

where $\varepsilon(n)>0$ and $\lim _{n \rightarrow \infty} \varepsilon(n)=0$.

Finally, the following lemma allows us to apply Lemma 4.

Lemma 8 [6, Lemma 3.1] Let $\mathcal{S}$ be a set of $n$ disjoint unit spheres in $\mathbb{R}^{d}$ with two switched pairs $(A, B)$ and $(C, D)$, for $n$ large enough. Then there are two line transversals $\ell$ and $\ell^{\prime}$ of $\mathcal{S}$ such that $\ell$ stabs the four spheres in the order $A B C D$ and $\ell^{\prime}$ stabs them in the order $B A D C$, possibly after interchanging $A$ and $B$ and/or $C$ and $D$.

Theorem 9 A set $\mathcal{S}$ of $n$ disjoint unit spheres in $\mathbb{R}^{d}$, for $n$ large enough, has at most one switched pair and admits at most two different geometric permutations.

Proof. The second claim follows from the first by Lemma 6 . Assume there are two different switched pairs $(A, B)$ and $(C, D)$. By Lemma 8 there exist two line transversals $\ell$ and $\ell^{\prime}$ and four spheres $A, B, C, D$ in $\mathcal{S}$ such that $\ell$ stabs them in the order $A B C D$ and $\ell^{\prime}$ stabs them in the order $B A D C$. Choosing $n$ large enough, we have by Lemma 7

$$
d(g(A, B), g(C, D)) \geq \sqrt{2}-1 / 5 .
$$

By Lemma 4, we also have

$$
d(g(A, B), g(C, D))<1+1 / 5<\sqrt{2}-1 / 5,
$$

a contradiction. The theorem follows.

\section{Acknowledgements}

We thank Hervé Brönnimann for introducing the problem to us. Part of this research was done during the Second McGill-INRIA Workshop on Computational Geometry in Computer Graphics at McGill Bellairs Research Institute. We wish to thank the institute for its hospitality, and especially Gwen, Bellair's chef, for her inspiring creations.

\section{References}

[1] A. Asinowski. Common transversals and geometric permutations. Master's thesis, Technion IIT, Haifa, 1998.

[2] H. Edelsbrunner and M. Sharir. The maximum number of ways to stab $n$ convex nonintersecting sets in the plane is $2 n-2$. Discrete Comput. Geom., 5:35-42, 1990.

[3] J. E. Goodman, R. Pollack, and R. Wenger. Geometric transversal theory. In J. Pach, editor, New Trends in Discrete and Computational Geometry. Algorithms and Combinatorics, vol. 10, pages 163-198. Springer-Verlag, 1993.

[4] Y. Huang, J. Xu, and D. Z. Chen. Geometric permutations of high dimensional spheres. In Proc. 12th ACM-SIAM Sympos. Discrete Algorithms, pages 244-245, 2001.

[5] M. Katchalski, T. Lewis, and A. Liu. The different ways of stabbing disjoint convex sets. Discrete Comput. Geom., 7:197-206, 1992.

[6] M. Katchalski, S. Suri, and Y. Zhou. A constant bound for geometric permutations of disjoint unit balls. Discrete $\mathscr{G}$ Computational Geometry, 29:161-173, 2003. 
[7] M. J. Katz and K. R. Varadarajan. A tight bound on the number of geometric permutations of convex fat objects in $\mathbb{R}^{d}$. Discrete Comput. Geom., 26:543-548, 2001.

[8] S. Smorodinsky, J. S. B. Mitchell, and M. Sharir. Sharp bounds on geometric permutations for pairwise disjoint balls in $\mathbb{R}^{d}$. Discrete Comput. Geom., 23:247-259, 2000.

[9] S. J. Teller and C. H. Séquin. Visibility preprocessing for interactive walkthroughs. Comput. Graph., 25:61-69, July 1991. Proc. SIGGRAPH '91.

[10] R. Wenger. Upper bounds on geometric permutations for convex sets. Discrete Comput. Geom., 5:27-33, 1990.

[11] R. Wenger. Helly-type theorems and geometric transversals. In J. E. Goodman and J. O'Rourke, editors, Handbook of Discrete and Computational Geometry, chapter 4, pages 63-82. CRC Press LLC, Boca Raton, FL, 1997. 